\title{
GeOMETRICAL Optics APPLIED TO 1D Site RESPONSE OF INHOMOGENEOUS SOIL DEPOSITS
}

A NON-PEER-REVIEWED PREPRINT UPLOADED TO ENGRXIV

\author{
Joaquin Garcia-Suarez \\ Graduate Aerospace Laboratories \\ California Institute of Technology \\ California, CA 91125 \\ ajgarcia@caltech.edu
}

\author{
Elnaz Seylabi \\ Civil and Enviromental Engineering \\ University of Nevada, Reno \\ Nevada, NV 89557 \\ elnaze@unr.edu
}

\author{
Domniki Asimaki \\ Mechanical and Civil Engineering \\ California Institute of Technology \\ California, CA 91125 \\ asimaki@caltech.edu
}

February 17, 2020

\begin{abstract}
The technique referred as Geometrical Optics entails considering the wave propagation in a heterogeneous medium as if it happened with infinitely small wavelength. This classic simplification allows to obtain useful approximate analytical results in cases where complete description of the waveform behavior is virtually unattainable, hence its wide use in Physics. This approximation is also commonly termed Ray Theory, and it has already been thoroughly applied in Seismology. This text presents an application of Geometrical Optics to 1D Site Response (1DSR): it is used herein to, first, explain and elucidate the generality of some previous observations and results; second, to partially settle an open question in 1DSR, namely "what are the equivalent homogeneous properties that yield the same response, in terms of natural frequencies and resonance amplitude, for a certain inhomogeneous site?", provided few assumptions.
\end{abstract}

Keywords Geotechnical Engineering · Site Response Analysis · Geometrical Optics · Ray Theory

\section{Introduction}

Site conditions and soil layering have paramount influence on seismic ground motions [Benuska, 1990]. The evolution of mechanical properties in the soil can induce a concentration of seismic energy in the softer layers [Towhata, 1996], which usually are those closer to the surface, and in turn translates into higher risk for man-made above-ground and underground structures.

A number of scholars have been occupied with studying the modification of ground seismic response due to the presence of inhomogeneous stratification in the soil by means of simplified one dimensional (1D) wave propagation models under the assumption of linear-elastic medium [Dobry et al., 1971, Gazetas, 1982, Vrettos, 2013, Vrettos, 1990, Zhao, 1997, Afra and Pecker, 2002, Travasarou and Gazetas, 2004, Semblat and Pecker, 2009], which happens to be suitable if

1) the material response is linear-elastic (there is an absence of either non-linear material response or large deformations),

2) the soil is horizontally layered in depth (thus the problem reduces to one-dimensional, vertical, wave propagation),

3) SH waves moving vertically conform the excitation,

4) material damping is frequency-independent.

The customary approach focuses on finding steady-state solution in frequency domain, which happens to be the dominant component of the response, except under some specific circumstances [Sarma, 1994], and it is also known to provide a good approximation to Ratios of Response Spectra (RRS) [Dobry et al., 1992, Joyner et al., 1992]. This 
simple approach has been favored in seismic building codes [Urzúa et al., 2017], even for sites displaying strong non-linear response [Ordaz and Meli, 2004], as a first step towards a more realistic assessment.

Although the problem can be solved analytically for some types of $c_{s}(y)$, a preferred approach is to define an equivalent uniform shear-wave (also termed "effective velocity") but how to define this parameter has remained an open debate. In particular, the usage of the harmonic average of the velocity distribution has been an option pursued since the 70s [Madera, 1970]. Despite the fact that this average overestimates (underestimates) the fundamental frequency of the site in cases having surface-to-bedrock decreasing (increasing) stiffness [Urzúa et al., 2017], it has been known [Zhao, 1997] to yield excellent estimates of the higher mode natural frequencies in sites having continuous profile given by the so-called "generalized parabola" [Rovithis et al., 2011]. A mathematical explanation of this fact was provided through recourse to the asymptotic behavior (for large frequencies) of the exact solution by the authors of this manuscript: it was found that in the high-frequency limit (corresponding to the resonance mode as high as desired) the exact equivalent velocity was indeed the harmonic mean of the profile.

This text seeks to fit the aforementioned result within the general theory of ray approximation for wave propagation inhomogeneous media [Kravtsov and Orlov, 1990] and to generalize the previous result, delivering a clear answer to the question "when should one use the harmonic mean of the shear wave velocity distribution as equivalent homogeneous velocity to calculate resonance modes in 1DSR?", provided the profile fulfills two straightforward conditions.

Answering the prior question can have important consequences, since equivalent velocity in terms of harmonic average is currently a widely-used parameter when it comes to account for soil heterogeneity, both in Site Response Analisys proper (see for instance the definition of $V_{s 30}$ in ASCE 7/16 [ASCE, 2016] or the discussion in [Urzua, 1974]) and Soil-Structure Interaction [NIST, 2012].

\subsection{Governing equation}

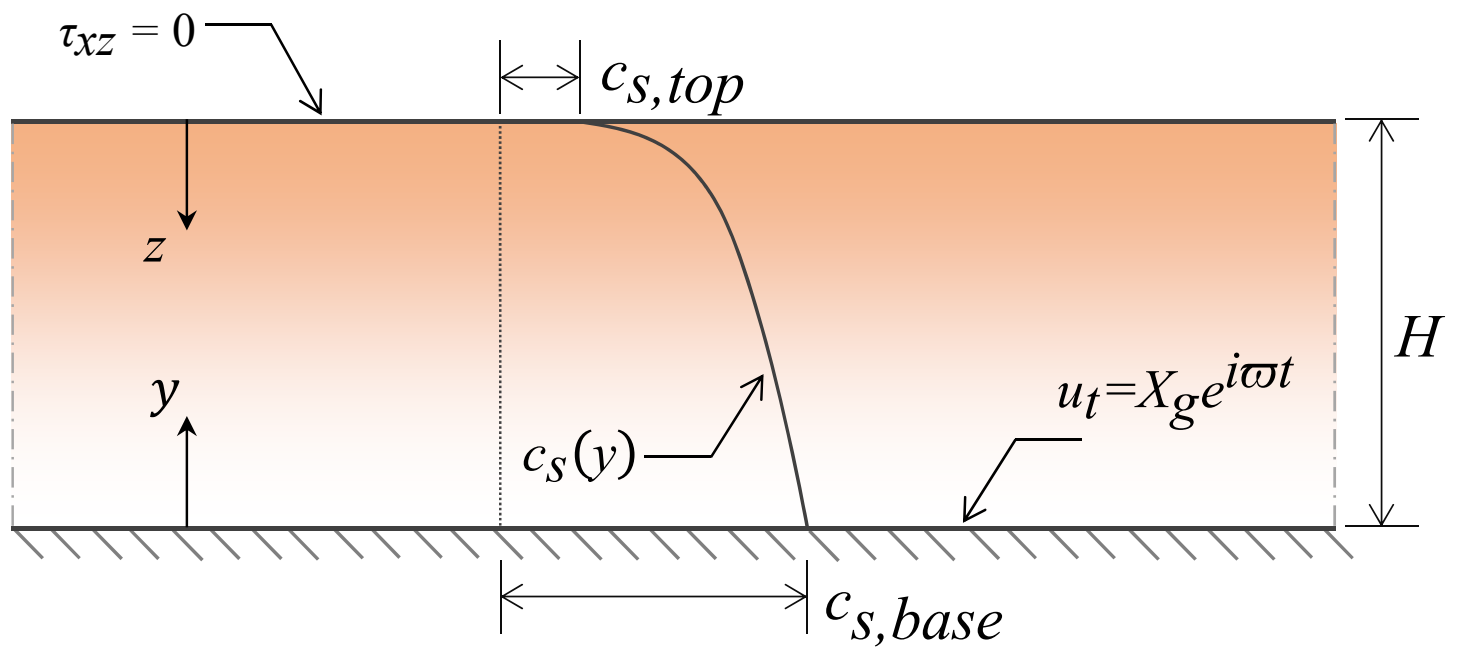

Figure 1: Scheme of the system considered in this study.

The harmonic wave-propagation problem to be considered, in terms of the total displacement amplitude $\hat{u}_{t}$, is [Rovithis et al., 2011]

$$
\frac{d}{d z}\left[\mu(z) \frac{d \hat{u}_{t}}{d z}\right]+\rho \varpi^{2} \hat{u}_{t}=0 \quad \text { s.t. } \quad \hat{u}_{t}(z=H)=X_{g} \quad,\left.\quad \frac{d \hat{u}_{t}}{d z}\right|_{z=0}=0,
$$

where $z$ is the coordinate stretching from the free surface to the rigid base, $\rho$ represents the density of the soil (which is assumed to be constant), the seismic S-wave excitation is modeled as a uniform displacement at the soil base $X_{g} e^{i \varpi t}$, and $\mu(z)$ represents the variation of shear modulus with depth. Damping is not shown explicitly in eq. (1) as it is not of the viscous type but hysteretic, hence the shear modulus is interpreted as an storage modulus (real number) plus a loss modulus (imaginary number) as $\mu(z) \rightarrow \mu(z)\left(1+\mathrm{i} \delta_{d}\right)$, where $\delta_{d}$ is referred as coefficient of material damping. As per usual arrangement, the density is taken as constant throughout the deposit.

For the sake of clarity, let us restate that the time variation comes given by $e^{i \varpi t}$, hence both $X_{g}$ and $\hat{u}_{t}$ are the amplitudes that accompany the phase: $X_{g}$ is a positive real number, whereas $\hat{u}_{t}$ represents a complex number whose modulus is 
the total displacement magnitude and whose argument represents the time shift between load (input) and displacement (response, output).

\subsection{Previous results}

In a previous work [Garcia-Suarez et al., 2020], the authors focused on a specific, commonly used, distribution of S-wave velocity, the so-called "generalized parabola" (density is assumed to be constant):

$$
\frac{c_{s}(z)}{c_{s, \text { base }}}=\sqrt{\frac{\mu(y)}{\mu_{\text {base }}}}=\left(b+(1-b) \frac{z}{H}\right)^{n}=\left(1+\left(\beta^{1 / n}-1\right) \frac{y}{H}\right)^{n},
$$

where $y=H-z$ is the complementary depth coordinate, $b=\beta^{1 / n}=\left(c_{s, t o p} / c_{s, b a s e}\right)^{1 / n}$ and $n$ is termed "inhomogeneity factor" (also referred to as "inhomogeneity parameter" [Dakoulas and Gazetas, 1985]). The parameter $b$ controls the maximum variation of $c_{s}$, which in this case always corresponds to top-to-bottom or vice versa, since the function only allows for monotonic evolution. The dimensionless expression of the problem, in terms of relative displacements, was used to re-expresses (1) in the following manner:

$$
\frac{d}{d \eta}\left[f^{2}(\eta) \frac{d \tilde{u}}{d \eta}\right]+r^{2} \tilde{u}=1, \quad \text { s.t. } \quad \tilde{u}(\eta=0)=0 \quad,\left.\quad \frac{d \tilde{u}}{d \eta}\right|_{\eta=1}=0
$$

where $f(\eta)=f(y / H)=c_{s}(y / H) / c_{s, b a s e}, \eta=y / H, r=\varpi H / c_{s, \text { base }}$ and $\tilde{u}=c_{s, b a s e}^{2} \hat{u} /\left(-\varpi^{2} X_{g} H^{2}\right), \hat{u}=X_{g}-\hat{u}_{t}$ being the amplitude of the relative displacement (relative to the base movement). $f(\eta)$ was assumed to be given by eq. (2) for all the developments carried out in the aforementioned work.

By analyzing the asymptotic behavior of the exact solution to (3) [Rovithis et al., 2011], the authors found that the base-to-top dynamic amplification could be expressed as

$$
A(\beta, n, r)=\frac{\sqrt{c_{s, b a s e} / c_{s, t o p}}}{\cos \left(\frac{\varpi}{c_{s, e q} / H}\right)}+\mathcal{O}\left(\frac{c_{s, e q} / H}{\varpi}\right),
$$

with $c_{s, e q}$ given by

$$
c_{s, e q}=H\left(\int_{0}^{H} \frac{d y}{c_{s}(y)}\right)^{-1},
$$

that is, the harmonic mean of the S-wave velocity distribution over the deposit. Since the "error" term in eq. (4), $\mathcal{O}(. .$.$) ,$ decays as the inverse of the frequency, its magnitude is guaranteed to be negligible if $\varpi>>c_{s, e q} / H$, hence retaining the first addend would be enough as to approximate the overall response in this regime. Likewise, in this regime the resonant peaks behaved as it the system was homogeneous having an equivalent material damping given by

$$
\delta_{d, e q}=\delta_{d} \sqrt{c_{s, t o p} / c_{s, \text { base }}} .
$$

Bear in mind, throughout this whole document, that any result concerning "high excitation frequency" could also be expressed in terms of "long wavelengths" of wave propagating within the soil.

The qualitative behavior put forward by eqs. (4) and (5) had been established previously [Zhao, 1997], but it had not been justified mathematically in terms of asymptotic behavior of the exact solution.

The previous result, on one hand, provided a simple estimate of the frequency response, in terms of amplitude and natural frequencies, and, on the other one, entailed the possibility of unequivocally defining a "homogeneous-equivalent" properties so as to obtain a response similar to the one of the exact solution of (3) in the high-frequency regime, provided the $S$-wave velocity distribution being furnished by eq. (2).

The obvious next move was to ascertain the scope of these results, verifying under what conditions they could be generalized to other profiles $f(\eta)$. This text is occupied with exactly that task.

\section{Application of Geometrical Optics}

For the purpose of generalizing the aforementioned results, only two requirements will be demanded of the function $f(\eta)$ : 
1) The profile is continuous and bounded with continuous and bounded first derivative, mathematically: $f(y) \in$ $C^{1}([0, H])$. This precludes considering layered profiles with sharp transitions (inter-layer impedance).

2) Only positive values of velocity are allowed, that is, $f(y)>0 \quad \forall y \in[0, H]$. This assumption prevents considering the case of zero stiffness at the free-surface [Rovithis et al., 2011] (this case, it will be shown, can be considered as a generalization of the results to be obtained though).

It is to be proved that an expression equivalent to eq. (4) holds for any distribution $f(\eta)$ verifying just these two conditions.

To do so, it turns out to be more convenient re-adopting the formulation in terms of total displacements (1), which, in dimensionless form reads

or

$$
\frac{d}{d \eta}\left(f^{2}(\eta) \frac{d \tilde{u}_{t}}{d \eta}\right)+r^{2} \tilde{u}_{t}=0,
$$

$$
f^{2}(\eta) \frac{d^{2} \tilde{u}_{t}}{d \eta^{2}}+2 f(\eta) f^{\prime}(\eta) \frac{d \tilde{u}_{t}}{d \eta}+r^{2} \tilde{u}_{t}=0,
$$

where in this case the non-dimensional total displacement is $\tilde{u}_{t}=\hat{u}_{t} / X_{g}$, and all the other dimensionless parameters and variables have been presented earlier. The boundary conditions of (1) are, now in dimensionless form,

$$
\tilde{u}_{t}(\eta=0)=1 \quad \text { and }\left.\quad \frac{d \tilde{u}_{t}}{d \eta}\right|_{\eta=1}=0 .
$$

Recall the high-frequency regime represents the focus of this work; such a regime corresponds to $r=\varpi H / c_{s, b a s e} \gg 1$, therefore $\epsilon=1 / r \ll 1$ may be considered as a small parameter [Simmonds and Mann, 2013]. Thus, rewrite eq. (7b) multiplying by $\epsilon^{2}$, and also dividing by $f^{2}(\eta)=0$ :

$$
\epsilon^{2} \frac{d^{2} \tilde{u}_{t}}{d \eta^{2}}+2 \epsilon^{2} \frac{f^{\prime}(\eta)}{f(\eta)} \frac{d \tilde{u}_{t}}{d \eta}+\frac{1}{f^{2}(\eta)} \tilde{u}_{t}=0,
$$

and, for the sake of brevity, call $q(\eta)=1 / f^{2}(\eta)$ (see that this function is always positive and bounded based on the restrictions of positive and non-zero wave velocity) and $m(\eta)=f^{\prime}(\eta) / f(\eta)$ (finite and positive too)

$$
\epsilon^{2} \frac{d^{2} \tilde{u}_{t}}{d \eta^{2}}+2 \epsilon^{2} m(\eta) \frac{d \tilde{u}_{t}}{d \eta}+q(\eta) \tilde{u}_{t}=0 .
$$

This is a singular perturbation problem with spatially-changing coefficients. The classic WKB method [Bender and Orszag, 2013] is the tool-of-choice to find an approximate solution. See that the condition $f(\eta) \neq 0$ $\forall y \in[0, H]$ is critical as it precludes the existence of turning points [Bender and Orszag, 2013].

In a nutshell, the WKB method advocates the use of the "ansatz"

$$
\tilde{u}_{t}(\eta) \sim \exp \left(\frac{1}{\delta} \sum_{n=0}^{\infty} \delta^{n} S_{n}(\eta)\right)
$$

in eq. (9b). A painstaking derivation can be found in the appendix at the end of this text. Let us proceed to state the main results that utilizing the method yields:

$$
S_{0}(\eta)= \pm i \int_{1}^{\eta} \sqrt{q\left(\eta^{\prime}\right)} d \eta^{\prime}= \pm i \int_{1}^{\eta} \frac{d \eta^{\prime}}{f\left(\eta^{\prime}\right)},
$$

recall that $q(\eta)=1 / f^{2}(\eta)=\left(c_{s, \text { base }} / c_{s}(y)\right)^{2}$; moreover

$$
S_{1}(\eta)=\mathcal{C}+\ln \left(f(\eta)^{-1 / 2}\right),
$$

where $\mathcal{C}$ represents a constant. Combining this two contributions of the phase (the only ones that do not vanish in the high-frequency limit) one obtains the physical optics approximation, which for the base-to-top amplification yields

$$
A(\varpi)=\frac{f(\eta=1)^{-1 / 2}}{\cos \left(\frac{\varpi H}{c_{s, e q}}\right)}=\frac{\sqrt{c_{s, \text { base }} / c_{s, \text { top }}}}{\cos \left(\frac{\varpi H}{c_{s, e q}}\right)},
$$

where $c_{s, e q}$ is the harmonic mean defined in eq. (5). This is the same expression as eq. (4), neglecting the "error" terms, therefore this result implies that the expression is valid for any profile abiding by the two constraints enunciated at the beginning of the section, regardless of other ancillary features. It is instructive to note that utilizing just $S_{0}$ yields the denominator and the harmonic mean homogeneous-equivalent velocity whereas including $S_{1}$ adds the amplitude correction associated to the heterogeneity.

The formal validity of the approximation is discussed in the appendix. If one wants to display the damping factor, $\delta_{d}$, explicitly in eq. (13), just substitute $c_{s, e q}$ by $c_{s, e q} \sqrt{1+\mathrm{i} \delta_{d}}$. 


\subsection{Generalization to evolving density}

A similar result would have been obtained had we allowed the density to be a function of depth. Had we done so, considering $\mu=\mu(y)=\mu_{\text {base }} g(\eta), \rho=\rho(y)=\rho_{\text {base }} h(\eta)$ (hence $f(\eta)^{2}=g(\eta) / h(\eta)$ ), satisfying the same conditions as discussed for $f(\eta)$, the auxiliary coefficients $m(\eta)$ and $q(\eta)$ would have become

$$
\begin{aligned}
m(\eta) & =\frac{1}{2} \frac{g^{\prime}(\eta)}{g(\eta)} \\
q(\eta) & =\frac{h(\eta)}{g(\eta)}
\end{aligned}
$$

These expressions yield a generalization of eq. (13):

$$
A(\varpi)=\frac{(h(\eta=1) g(\eta=1))^{-1 / 4}}{\cos \left(r \int_{0}^{1} \frac{d \eta}{f(\eta)}\right)},
$$

reversing the non-dimensionalization,

$$
A(\varpi)=\left(\frac{\mu_{\text {base }} \rho_{\text {base }}}{\mu_{\text {top }} \rho_{\text {top }}}\right)^{1 / 4} \sec \left(\frac{\omega H}{c_{s, e q}}\right)
$$

where $c_{s, e q}$ is still being given by the harmonic mean of $f(\eta)=\sqrt{g(\eta) / h(\eta)}$, eq. (5), and now the change of amplitude depends on the base-to-top relative evolution both of stiffness and the density and density (observe that eq. (13) simply corresponds to $\rho_{\text {base }}=\rho_{\text {top }}$ ).

\section{Physical significance}

\subsection{Interpretation}

The physical interpretation of these results is straightforward once they are framed in terms of wavelength: high frequencies entail relatively short wavelengths, short with respect to $H$, propagating inside the material bulk. Short wavelengths tend to "smooth out" those spatial features of the medium larger than themselves, and thus, one may say, the wave does not "see" any shift in stiffness and it behaves as if there was only uniform soil all around, while long wavelengths "see" every stiffness alteration, and hence it tends to displace "chunks" of truly heterogeneous soil.

The reason for obtaining the harmonic mean instead of the arithmetic mean derive from the fact that in this problem the travel distance is kept constant, not the travel time. Let us illustrate this point carefully: the stratum in Figure 1 can be conceptualized as a series of infinitely many slices each with the same infinitesimal thickness, $h=H / N$ where the number of slices, $N$, tends to infinity, hence we calculate the average velocity of the propagating wave as the ratio between the total travel distance over the total time that the journey takes

$$
c_{s, \text { mean }}=\frac{\sum_{i=1}^{N} h_{i}}{\sum_{i=1}^{N} T_{i}}=\frac{H}{\sum_{i=1}^{N}\left(H / N c_{s, i}\right)} \rightarrow \frac{1}{\int_{0}^{H} \frac{d(y / H)}{c_{s}(y)}}=c_{s, e q}
$$

as $N$ tends to infinity. The subscript $i$ denotes properties concerning the $i$-th discrete slice. This means that the proper average to consider in this kind of problem is the harmonic mean.

This interpretation is by no means original, it is simply an adaptation of well-known results in Classical Optics (i.e., propagation of light as a electromagnetic wave) which date back to the works of Fermat and Huygens.

\subsection{This result in the broad context of Ray Theory}

The previous results can be considered as the outcome of application of so-called ray approximation methods to the problem (1). These employ the short-wavelength (high-frequency) limit to analyze wave propagation phenomena (electromagnetic, acoustic or elastic). Ray methods are a powerful tool that has already been used in Elastodynamics; see for example the monograph by Prof. Achenbach and coworkers [Achenbach et al., 1982], wherein these methods are applied to the study of the effect of cracks over waves propagating within homogeneous elastic bodies.

The physical intuition behind these results has been common currency in the physics community, including seismology, see Aki and Richards' treaty on Quantitative Seismology [Aki and Richards, 2002], in particular, Chapter 4, section 
4, concerning ray methods as a means to consider seismic wave propagation in the heterogeneous earth. However, these appraisals had not been passed on to the geotechnical engineering community at large nor, it appears, explicitly spelled out in the mathematical terms of the WKB approximation applied to considerations of unidimensional propagation in a finite domain, although similar expansions are usually discussed in the geometrical optics literature [Achenbach et al., 1982, Kravtsov and Orlov, 1990], including the renowned Debye expansion, which does not rely on the definition of small parameters directly.

This intuition also allows to reply to the following question: "what does it happen when $f(y) \notin C^{1}([0, H])$ but $f(y) \in C^{0}([0, H])$ (continuous function, non-continuous first derivative) or even $f(y) \in C^{-1}([0, H])$ (non-continuous function)?". The reasoning that was followed in section 2 is forbidden since $f^{\prime}(\eta)$ cannot be defined. The getaway is found in Fermat's Principle: basically, Fermat's Principle states that the problem we are solving possesses a variational structure, and hence the equations we are solving are nothing but its corresponding Euler's equations [Gelfand et al., 2000]. If the functions describing the parameter evolution are not continuous enough, this may prevent us from defining the Euler's equations properly, but the underlying stationarity condition [Kravtsov and Orlov, 1990] remains in effect. The phenomenon at the intersection of different mediums can be described by an analogue of the Snell's law in Optics.

The only "exotic" feature that this specific instantiation introduces concerns where the inhomogeneity is localized. Normally, the wave propagates in a medium with varying refraction index, hence the elliptic operator that appears in Euler's equations is simply a Laplacian, which in the 1D-case boils down to a neat second derivative in space, $\partial^{2}(\cdot) / \partial y^{2}$, where the dot represents the dependent variable; in this case, at least part of the homogeneity is confined "within the operator" due to the change in stiffness of the medium, hence the operator one has to deal with is $\partial(\mu(y) \partial(\cdot) / \partial y) / \partial y$, hence this problem can be considered as an instantiation of Sturm-Liouville Theory [Weinberger, 2012].

In order to perform a one-to-one mapping between the classic theory and prior results, let us state that the first component to the phase, $S_{0}$ is equivalent to the eikonal function (is actually equal to it times an extra factor $\pm \mathrm{i}$ ), the refraction index corresponds to the inverse of the velocity distribution, and, just like the general theory foresees, the eikonal function is proportional to the integral of the refraction index over the arc-length of the trajectory followed by the ray [Kravtsov and Orlov, 1990], which in this case happen to be straight vertical lines. The second component of the phase, $S_{1}$, yields the leading-order approximation of the amplitude, which is provided by solving a transport equation. Moreover, the amplification factor in both eq. (16) and eq. (13) can be retraced to the ray divergence parameter, which is but a consequence of the energy flux conservation in the system. See [Kravtsov and Orlov, 1990] for details.

Before concluding this digression, let us mention, on one hand, that the case corresponding to $\delta_{d}=\delta_{d}(y) \ll 1$ can be assimilated into a classic scenario dubbed as medium with weak absorption, and, secondly, that the case of vanishing stiffness at the top of the layer can be understood as the presence of a caustic surface at the top of the stratum [Stavroudis, 2012].

\section{Comparison to numerical results}

Let us illustrate how and when the approximate formula eq. (13) agrees well with an exact solution that could still be obtained numerically for cases of complicated profiles. In order to illustrate this, two "toy" profiles shall be utilized.

\subsection{Increasing contrast}

First, let us consider the influence of sudden mechanical property changes on the fitness of the approximation. For such purpose, consider

$$
f(\eta)=1-\frac{3}{8}\left[1+\tanh \left(p\left(\eta-\frac{1}{3}\right)\right)\right] .
$$

This profile gives a contrast between base and top of $1 / 4$, where all the variation is concentrated at $\eta=1 / 3$. The parameter $p$ controls the gradient in that zone, the higher $p$ the quicker the evolution and the more sudden the change. In the limit $p \rightarrow \infty$ the function becomes an step function and the gradient is infinite, so the condition $f(\eta) \in C^{1}([0,1])$, upon which eq. (13) was obtained, stops holding. Three different cases have been considered using Mathematica [Wolfram, 2000], by numerically solving eq. (7b) for three increasing values of $p(5,10$, and 100).

We observe in Figure 2 how the approximate formula eq. (13) successfully captures the behavior in most of the frequency range, but the agreement worsens as the smooth change becomes a localized snap at $p=100$. This result illustrates how it behooves the gradient to remain bounded everywhere for the approximation to match the exact solution. 


\subsection{Profile reversal}

This time, let us consider a profile with reversals (softer regions in between stiffer ones). For this task, use

$$
f(\eta)=1+\Delta \sin \left(K \frac{\pi}{2} \eta\right),
$$

where $\Delta$ controls the amplitude of the reversals whereas $K$ does the same for the number of reversals that happen within the layer. This example is intended to confirm the lack of influence of the monotonicity of $f(\eta)$ over the result eq. (13).

On can acknowledge in Figure 3 that the number of oscillations, $K$, and their amplitude $\Delta$, in and of itself, do not influence the convergence of the approximation to the exact value, but the gradient, which in this case is linearly proportional to both $K$ and $\Delta$, is the parameter to control, and obviously the more turns within the finite layer and the acuter these are have a direct translation on the gradient magnitude. This explains the deterioration from the fist case to the last one. This display embodies the irrelevance of monotonicity requirements on the profile evolution for eq. (13) to hold. 

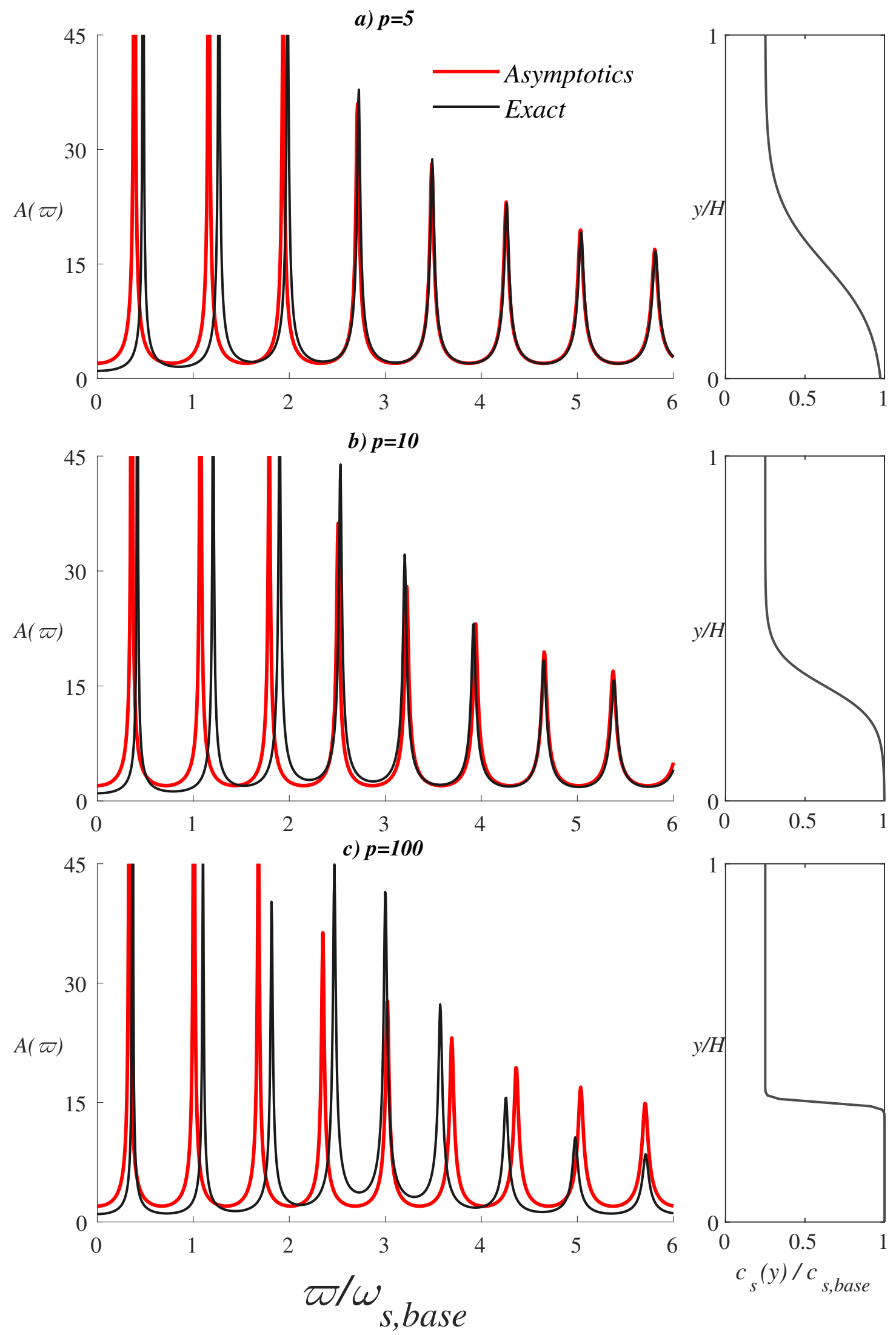

Figure 2: Base-to-top dynamic amplification $A(\varpi)$ (with corresponding vertical S-wave profile $c_{s}(y)$ ), comparison between numerical solution of eq. (1) (Exact) and eq. (13) (Asymptotics) 

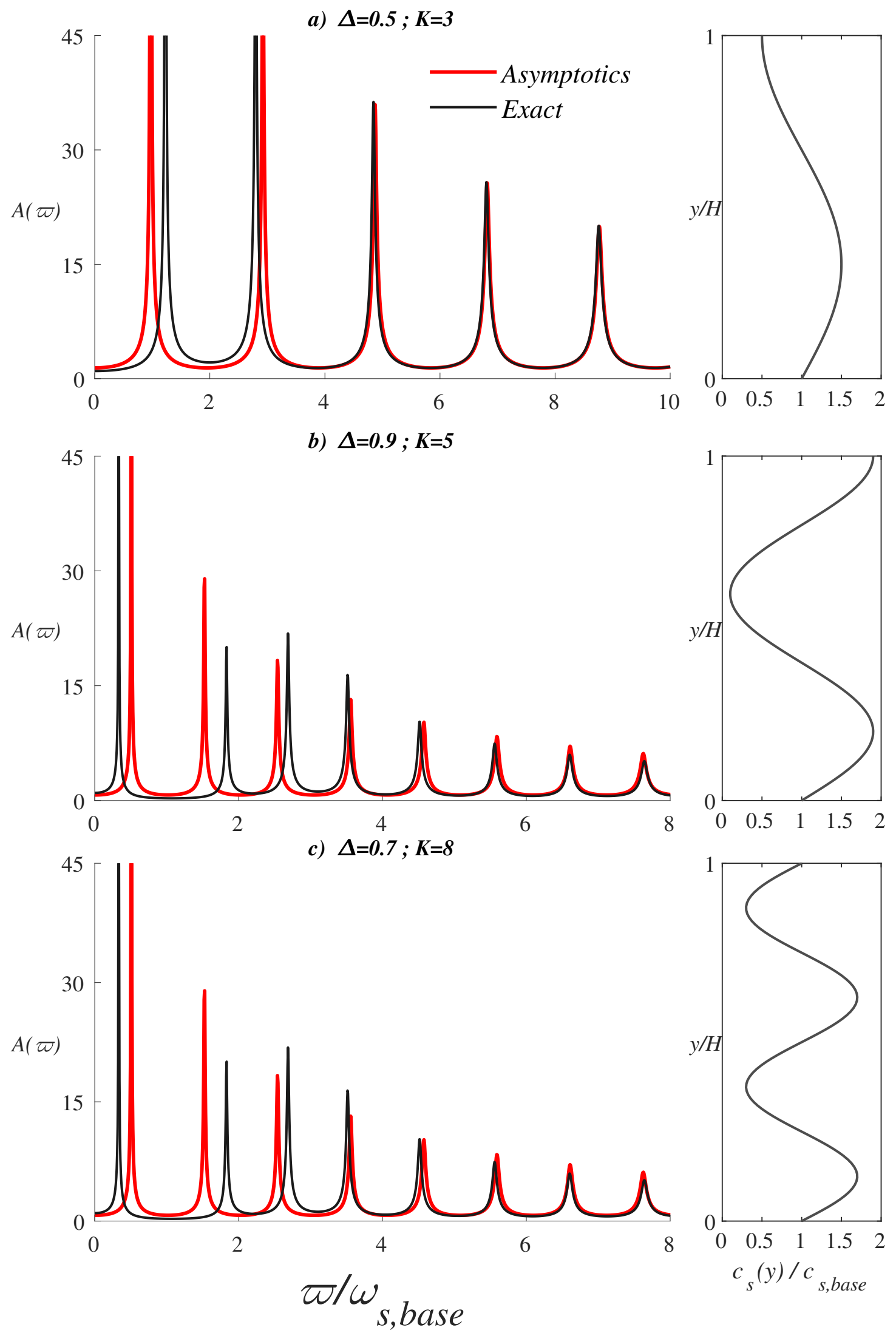

Figure 3: Base-to-top dynamic amplification $A(\varpi)$ (with corresponding vertical S-wave profile $c_{s}(y)$ ), comparison between numerical solution of eq. (1) (Exact) and eq. (13) (Asymptotics) 


\section{Implications for NIST and ASCE recommendations}

Current recommendations [NIST, 2012] by the National Institute of Standards and Technology (NIST) regarding Soil-structure Interaction (SSI) instruct the use of the harmonic mean of shear-wave velocity distribution, for any value of excitation frequency, when considering SSI (rocking, swaying and other phenomena) of foundations resting over heterogeneous soil.

This recommendation may be re-evaluated in light of the results displayed previously, as it appears to be unfitting to use the harmonic mean of the velocity distribution as the equivalent one for the whole range of excitation frequencies; such a value should be limited to specific combinations of frequency, soil mechanical properties $c_{s}$ and depth to bedrock $H$ so that $\varpi H / c_{s} \gg 1$.

When it comes to define equivalent homogeneous property to consider the amplitude of the fundamental mode in a 1D site response scenario that includes heterogeneity, our results indicate that using harmonic average to be unfitting except in cases having very minor heterogeneity. Other results published by the authors suggest, contrariwise, properties of the soil layer in contact with bedrock should be used as these control the fundamental response [Garcia-Suarez and Asimaki, 2019].

\section{Conclusions and future work}

\subsection{Conclusions}

This work has been occupied, chiefly, with a generalization of previous results obtained under the assumption of S-wave velocity profile abiding by eq. (2) [Garcia-Suarez et al., 2020] to any profile meeting two simple requirements: 1) continuity and boundedness of first derivative of $f(\eta), 2$ ) positive (non-zero) values of $c_{s}$ throughout the deposit, including at the free-surface. It has been shown that this result stems from a classical result in Physics, the so-called Geometrical Optics approximation.

These results also settle partially the debate over the equivalent-homogeneous properties that should be used to describe the response of inhomogeneous soil deposits overlying rigid bedrock: provided $\varpi H / c_{s, b a s e} \gg 1$ and the two constraints in Section 2 are satisfied, eq. (5) described the equivalent-homogeneous S-wave velocity, whereas the resonance peaks behave as if the material damping came given by eq. (6).

In spite of the application, if the amplitude as small as for the linear-elastic response assumption to remain in effect, the approximation eq. (13) may still be applied to a portion of the earthquake spectrum.

\subsection{Future work}

Since the approximation is not limited to finite layers with zero impedance at the bottom, the previous results could be extended to waves propagating vertically in a halfspace, hence generalizing the results to those scenarios wherein radiational damping [Kramer, 1996] has to be accounted for.

\section{Acknowledgments}

The authors are thankful to Prof. Michael Ortiz for his input regarding connections to Geometrical Optics Theory at large.

The format used in this preprint has been dispensed by Mr. George Kour through Overleaf under Creative Commons CC BY 4.0.

\section{References}

[Achenbach et al., 1982] Achenbach, J. D., Gautesen, A. K., and McMaken, H. (1982). Ray methods for waves in elastic solids: with applications to scattering by cracks.

[Afra and Pecker, 2002] Afra, H. and Pecker, A. (2002). Calculation of free field response spectrum of a nonhomogeneous soil deposit from bed rock response spectrum. Soil Dynamics and Earthquake Engineering, 22(2):157165.

[Aki and Richards, 2002] Aki, K. and Richards, P. G. (2002). Quantitative seismology.

[ASCE, 2016] ASCE (2016). Minimum design loads and associated criteria for buildings and other structures. 
[Bender and Orszag, 2013] Bender, C. M. and Orszag, S. A. (2013). Advanced mathematical methods for scientists and engineers I: Asymptotic methods and perturbation theory. Springer Science \& Business Media.

[Benuska, 1990] Benuska, L. (1990). Ground motion, loma prieta earthquake reconnaissance report. Earthquake Spectra, 6:25-80.

[Dakoulas and Gazetas, 1985] Dakoulas, P. and Gazetas, G. (1985). A class of inhomogeneous shear models for seismic response of dams and embankments. International Journal of Soil Dynamics and Earthquake Engineering, 4(4):166-182.

[Dobry et al., 1992] Dobry, R., Martin, G., Parra, E., and Bhattacharyya, A. (1992). Development of site-dependent ratios of elastic response spectra (rrs) and site categories for building seismic codes. In Proceedings NCEER, SEAOC, BSSC Workshop on Site Response During Earthquakes and Seismic Code Provisions, University of Southern California, Los Angeles, November, volume 18, page 20.

[Dobry et al., 1971] Dobry, R., Whitman, R. V., and Vinuesa, J. M. R. (1971). Soil properties and the one-dimensional theory of earthquake amplification. MIT Department of Civil Engineering, Inter-American Program.

[Garcia-Suarez and Asimaki, 2019] Garcia-Suarez, J. and Asimaki, D. (2019). Fundamental mode resonant amplitude of inhomogeneous soil deposits. engrXiv preprint engrXiv:- .

[Garcia-Suarez et al., 2020] Garcia-Suarez, J., Esmaeilzadeh, E., and Asimaki, D. (accepted, 2020). Seismic harmonic response of inhomogeneous soil: scaling analysis. Géotechnique.

[Gazetas, 1982] Gazetas, G. (1982). Vibrational characteristics of soil deposits with variable wave velocity. International journal for numerical and analytical methods in Geomechanics, 6(1):1-20.

[Gelfand et al., 2000] Gelfand, I. M., Silverman, R. A., et al. (2000). Calculus of variations. Courier Corporation.

[Joyner et al., 1992] Joyner, W., Fumal, T., and Glassmoyer, G. (1992). Empirical spectral response ratios for strong motion data from the 1989 loma prieta, california, earthquake. In Proceedings of 1992 NCEER/SEAOC/BSSC Workshop on Site Response during Earthquake and Seismic Code Provisions.

[Kramer, 1996] Kramer, S. L. (1996). Geotechnical earthquake engineering. in Prentice-Hall international series in civil engineering and engineering mechanics. Prentice Hall Inc., Englewood Cliffs, New Jersey.

[Kravtsov and Orlov, 1990] Kravtsov, Y. A. and Orlov, Y. I. (1990). Geometrical optics of inhomogeneous media. Geometrical Optics of Inhomogeneous Media by Yu. A. Kravtsov, Yu. I. Orlov Berlin, GR: Springer-Verlag, 1990.

[Madera, 1970] Madera, G. A. (1970). Fundamental period and amplification of peak acceleration in layered systems. MIT Department of Civil Engineering, Inter-American Program.

[NIST, 2012] NIST, N. (2012). Soil-structure-interaction for building structures (nist gcr 12-917-21). National Institute of Standards and Technology, Gaithersburg, MD, 20899.

[Ordaz and Meli, 2004] Ordaz, M. and Meli, R. (2004). Seismic design and codes in mexico. In Proc. 13th World Conf. on Earthquake Engineering.

[Rovithis et al., 2011] Rovithis, E., Parashakis, H., and Mylonakis, G. (2011). 1d harmonic response of layered inhomogeneous soil: Analytical investigation. Soil Dynamics and Earthquake Engineering, 31(7):879-890.

[Sarma, 1994] Sarma, S. (1994). Analytical solution to the seismic response of visco-elastic soil layers. Geotechnique, 44(2):265-275.

[Semblat and Pecker, 2009] Semblat, J. and Pecker, A. (2009). Waves and vibrations in soils. Earthquakes, traffic, shocks.

[Simmonds and Mann, 2013] Simmonds, J. G. and Mann, J. E. (2013). A first look at perturbation theory. Courier Corporation.

[Stavroudis, 2012] Stavroudis, O. (2012). The optics of rays, wavefronts, and caustics, volume 38. Elsevier.

[Towhata, 1996] Towhata, I. (1996). Seismic wave propagation in elastic soil with continuous variation of shear modulus in the vertical direction. Soils and Foundations, 36(1):61-72.

[Travasarou and Gazetas, 2004] Travasarou, T. and Gazetas, G. (2004). On the linear seismic response of soils with modulus varying as a power of depth: the maliakos marine clay. Soils and foundations, 44(5):85-93.

[Urzua, 1974] Urzua, A. (1974). Determinacion del periodo fundamental de vibracion del suelo. Master's Thesis in Soil Mechanics, University of Chile, Santiago, Chile.

[Urzúa et al., 2017] Urzúa, A., Dobry, R., and Christian, J. T. (2017). Is harmonic averaging of shear wave velocity or the simplified rayleigh method appropriate to estimate the period of a soil profile? Earthquake Spectra, 33(3):895915 . 
[Vrettos, 1990] Vrettos, C. (1990). Dispersive sh-surface waves in soil deposits of variable shear modulus. Soil Dynamics and Earthquake Engineering, 9(5):255-264.

[Vrettos, 2013] Vrettos, C. (2013). Dynamic response of soil deposits to vertical sh waves for different rigidity depth-gradients. Soil Dynamics and Earthquake Engineering, 47:41-50.

[Weinberger, 2012] Weinberger, H. F. (2012). A first course in partial differential equations: with complex variables and transform methods. Courier Corporation.

[Wolfram, 2000] Wolfram, S. (2000). The mathematica book, volume 4. Cambridge University Press Cambridge.

[Zhao, 1997] Zhao, J. (1997). Modal analysis of soft-soil sites including radiation damping. Earthquake engineering \& structural dynamics, 26(1):93-113.

\section{Appendix: Details on derivations}

As it was mentioned in the body of the paper, introducing the ansatz eq. (10) into eq. (9b) yields

$$
\epsilon^{2}\left[\frac{1}{\delta^{2}}\left(\sum_{n=0}^{\infty} \delta^{n} S_{n}^{\prime}(\eta)\right)^{2}+\frac{1}{\delta}\left(\sum_{n=0}^{\infty} \delta^{n}\left(S_{n}^{\prime \prime}(\eta)+2 m(\eta) S_{n}^{\prime}(\eta)\right)\right)\right]+q(\eta)=0,
$$

to leading order $(n=0)$ :

$$
\frac{\varepsilon^{2}}{\delta^{2}} S_{0}^{\prime 2}(\eta)+\frac{\varepsilon^{2}}{\delta}\left[2 S_{0}^{\prime}(\eta) S_{1}^{\prime}(\eta)+S_{0}^{\prime \prime}(\eta)+2 m(\eta) S_{0}^{\prime}(\eta)\right]+q(\eta)=0
$$

in the limit $\delta \rightarrow 0$, dominant balance imposes $\delta \sim \epsilon$, and therefore

$$
\frac{\varepsilon^{2}}{\delta^{2}} S_{0}^{\prime 2}(\eta)+q(\eta)={S^{\prime 2}}_{0}^{2}(\eta)+q(\eta)=0
$$

for such a limit. Then, by simple integration of eq. (22))

$$
S_{0}(\eta)=\int_{1}^{\eta} \sqrt{-q\left(\eta^{\prime}\right)} d \eta^{\prime}= \pm i \int_{1}^{\eta} \sqrt{q\left(\eta^{\prime}\right)} d \eta^{\prime}
$$

where $\eta^{\prime}$ represents a dummy integration variable.

Note also that eq. (10) turns into

$$
\tilde{u}(\eta) \sim \exp \left(\frac{1}{\epsilon} \sum_{n=0}^{\infty} \epsilon^{n} S_{n}(\eta)\right)
$$

and recalling that $r=1 / \epsilon$

$$
\tilde{u}(\eta) \sim \exp \left(r \sum_{n=0}^{\infty} \frac{S_{n}(\eta)}{r^{n}}\right)=\exp \left(r S_{0}(\eta)+S_{1}(\eta)+\frac{S_{2}(\eta)}{r}+\ldots\right) .
$$

Thus, to first-order approximation

$$
\tilde{u}(\eta)=A \exp \left(i r \int_{1}^{\eta} \sqrt{q\left(\eta^{\prime}\right)} d \eta^{\prime}\right)+B \exp \left(-i r \int_{1}^{\eta} \sqrt{q\left(\eta^{\prime}\right)} d \eta^{\prime}\right)
$$

which, after imposing boundary conditions to find $A, B$ boils down to

$$
\tilde{u}(\eta)=\frac{\cos \left(r \int_{\eta}^{1} \sqrt{q\left(\eta^{\prime}\right)} d \eta^{\prime}\right)}{\cos \left(r \int_{0}^{1} \sqrt{q\left(\eta^{\prime}\right)} d \eta^{\prime}\right)} .
$$

We could undo the non-dimensionalization at this point, but let us find the base-to-surface amplification, $A$, first, and then do so:

$$
A(\varpi)=\frac{u(y=H)}{X_{g}}=\tilde{u}(\eta=1)=\frac{1}{\cos \left(r \int_{0}^{1} \sqrt{q\left(\eta^{\prime}\right)} d \eta^{\prime}\right)}=
$$


now remove dimensionless variables and parameters, in particular, take into account that $\sqrt{q(\eta)}=1 / f(\eta)=$ $1 /\left(c_{s}(y) / c_{s, \text { base }}\right)$

$$
=\frac{1}{\cos \left(r \int_{0}^{1} \frac{d \eta^{\prime}}{f\left(\eta^{\prime}\right)}\right)}=\frac{1}{\cos \left(\varpi \int_{0}^{H} \frac{d y}{c_{s}(y)}\right)}=\frac{1}{\cos \left(\frac{\varpi H}{c_{s, e q}}\right)},
$$

where

$$
c_{s, e q}=H\left(\int_{0}^{H} \frac{d y}{c_{s}(y)}\right)^{-1},
$$

happens to be the harmonic mean of the function $c_{s}(y)$ in the interval $[0, H]$.

This is the part of the recursion concerning $\epsilon^{0}$. Now we can proceed to find the next term in the approximation, $\epsilon^{1}$ :

$$
\begin{array}{r}
2 S_{0}^{\prime}(\eta) S_{1}^{\prime}(\eta)+S_{0}^{\prime \prime}(\eta)+2 m(\eta) S_{0}^{\prime}(\eta)=0, \\
2 S_{1}^{\prime}(\eta)+\frac{S_{0}^{\prime \prime}(\eta)}{S_{0}^{\prime}(\eta)}+2 m(\eta)=0,
\end{array}
$$

recall $m(\eta)=f^{\prime}(\eta) / f(\eta)$, and then integrate (let $\mathcal{C}$ be a constant):

$$
\begin{aligned}
2 S_{1}(\eta)+\ln \left(S_{0}^{\prime}(\eta)\right)+2 \ln f(\eta) & =\mathcal{C}, \\
2 S_{1}(\eta)+\ln \left(S_{0}^{\prime}(\eta) f^{2}(\eta)\right) & =\mathcal{C},
\end{aligned}
$$

see that, from eq. (22)), $S_{0}^{\prime}(\eta)=\sqrt{-q(x)}=i / f(\eta)$

$$
\begin{aligned}
2 S_{1}(\eta)+\ln (i)+\ln (f(\eta)) & =\mathcal{C}, \\
2 S_{1}(\eta)+i \frac{\pi}{2}+\ln (f(\eta)) & =\mathcal{C}, \\
S_{1}(\eta) & =\frac{\mathcal{C}}{2}-i \frac{\pi}{4}+\ln \left(f(\eta)^{-1 / 2}\right) .
\end{aligned}
$$

Thus, adding this new term into eq. (24b) yields

$$
\tilde{u}(\eta)=f(\eta)^{-1 / 2} \frac{\cos \left(r \int_{\eta}^{1} \sqrt{q\left(\eta^{\prime}\right)} d \eta^{\prime}\right)}{\cos \left(r \int_{0}^{1} \sqrt{q\left(\eta^{\prime}\right)} d \eta^{\prime}\right)}
$$

and hence the amplification

$$
A(\varpi)=\frac{\left(c_{s, t o p} / c_{s, \text { base }}\right)^{-1 / 2}}{\cos \left(\frac{\varpi H}{c_{s, e q}}\right)} .
$$

\section{Validity conditions}

The approximation to be verified all over the layer it is necessary for the parameters to verify

$$
r \gg \frac{\ln \left(f(\eta)^{-1 / 2}\right)}{\int_{0}^{\eta} \frac{d \eta}{f(\eta)}}, \quad \forall \eta \in[0,1] \quad \text { as } \quad r \rightarrow \infty .
$$

Note that, however large the left-hand side is, one can always define a high-enough frequency so that the inequality is verified.

Moreover, from a "small-parameter standpoint", eq. (13) is always valid for as long as $f^{\prime}(\eta) / f(\eta)$ is not as large as to compensate the presence of the small parameter $\epsilon$ in eq. (9b)): in mathematical terms, it must be verified that

$$
\epsilon^{2}\left|\frac{f^{\prime}(\eta)}{f(\eta)}\right| \ll 1,
$$

or inverting $\epsilon$

$$
\left|\frac{f^{\prime}(\eta)}{f(\eta)}\right| \ll r^{2}
$$


See that this local condition does not when there are either sudden jumps in the velocity profile, as it happens in those cases $f^{\prime}(\eta) \rightarrow \infty$, viz. when the gradient is large (potentially infinite), or complementarily when the derivative at some point cannot be defined (when for example the profile presents a kink). Nevertheless, as long as the gradient, and hence the velocities, remains bounded there is always a sufficiently high frequency at which point the approximation starts being valid. It is interesting to note that the sign of $f^{\prime}(\eta)$ does not affect the result, for the approximation to be adequate only the magnitude of the gradient does matter while its sign is irrelevant, so the result holds if the velocity profile is globally or locally increasing or decreasing.

\section{Generalization}

An even-more-general result can be obtained: using this small parameters framework one can show that actually a similar result holds even for the case wherein both stiffness and density change across the layer.

Suppose $\rho=\rho_{\text {base }} h(\eta)$, whereas $\mu=\mu_{\text {base }} j(\eta)$, where both $j(\eta)$ and $j(\eta)$ are positive and bounded functions in the interval and also belong in $C^{1}([0,1])$. The corresponding counterpart of eq. (9a) is

$$
\epsilon^{2} \frac{d^{2} \tilde{u}_{t}}{d \eta^{2}}+\epsilon^{2} \frac{j^{\prime}(\eta)}{j(\eta)} \frac{d \tilde{u}_{t}}{d \eta}+\frac{h(\eta)}{j(\eta)} \tilde{u}_{t}=0
$$

thus redefining $m(\eta)=j^{\prime}(\eta) / 2 j(\eta)$ and $q(\eta)=h(\eta) / j(\eta)$ yields a new eq. (9b) again, which, repeating the procedure,

$$
A(\varpi)=\frac{\left(\frac{\rho_{\text {top }} \mu_{\text {top }}}{\rho_{\text {base }} \mu_{\text {base }}}\right)^{1 / 4}}{\cos \left(\frac{\varpi H}{c_{s, e q}}\right)},
$$

where $c_{s, e q}$ is still given by the harmonic mean of the point-wise defined shear-wave velocity distribution, as originally given by $f(\eta)=\sqrt{\mu(\eta) / \rho(\eta)}$. 